\title{
A Constant Acoustic Impedance Mount for Sheet-Type Specimens in the Ten- sile Split-Hopkinson Bar
}

\author{
Georg. C. Ganzenmüller ${ }^{1,2, *}$, Timo Langhof ${ }^{1}$, and Stefan Hiermaier ${ }^{1,2}$ \\ ${ }^{1}$ Albert-Ludwigs University of Freiburg, Department for Sustainable Systems Engineering, INATECH, Germany \\ ${ }^{2}$ Fraunhofer Ernst-Mach Institute for High-Speed Dynamics, EMI, Freiburg, Germany
}

\begin{abstract}
This paper addresses a problem well-known amongst practitioners of the Split-Hopkinson Tension Bar method: attaching a flat test specimen made from sheet material to the cylindrical input- and output bars. To date, slotting the bar ends and gluing the specimens into these ends with high-strength adhesives is the gold standard. However, this approach is not universally applicable because some materials are difficult to bond, and the adhesion surface is limited by the bar diameter, meaning that only small width specimens can be tested. In contrast, the hitherto published mechanical clamping mechanisms typically introduce excessive additional mass into the Split-Hopkinson system which detrimentally affects wave propagation and thus causes errors in the stress-strain signals. We circumvent this problem by designing a mechanical clamping device which has the same acoustic impedance as the bar material and is suitable to securely attach specimens with a width larger than the bar diameter. The benefits of our new clamping device are demonstrated by reporting tensile stress-strain data for Polycarbonate at high strain rates. The data is free from unwanted oscillations and enables accurate determination of dynamic strength and stiffness.
\end{abstract}

\section{Introduction}

The Split-Hopkinson Tension Bar (SHTB) allows to perform dynamic material characterization in the strain rate regime of approximately $10^{2}-10^{4} \mathrm{~s}^{-1}$. This experimental technique is therefore well suited to cover strain rates in excess of $10^{1} \mathrm{~s}^{-1}$, where servo-hydraulic universal testing machines start to exhibit pronounced oscillations in the measured stress-strain signals due to multiple reflections of elastic waves [1]. The SHTB avoids these unwanted reflections by passing only a single, well defined incident elastic wave pulse through a co-linear arrangement of striker, input bar, test specimen, and output bar. The requirement of using only a single wave imposes an upper limit on the duration of the usable experiment time, which is determined by the lengths of striker and input / output bars. The analogue of the universal testing machine's crosshead speed is the striker velocity, which is limited by the strength of the striker and the strength of the bar material. In practice, maximum pulse durations on the order of $1 \mathrm{~ms}$ and striker velocities up to $40 \mathrm{~m} / \mathrm{s}$ are currently possible [2-4]. From these numbers, the achievable strain rates can be calculated for a given test specimen geometry. In addition to the finite strain rate range, a major additional constraint exists for the SHTB technique which renders it less universally applicable than the universal testing machine itself. In the analysis of the experiment, it is assumed that there are only three different generalized acoustic impedances present in the system: (i) the constant impedance of the input bar, $z_{i}=A_{i} c_{i} \rho_{i}(A$

\footnotetext{
*e-mail: georg.ganzenmueller@inatech.uni-freiburg.de
}

being cross section area, $c$ elastic wave speed, and $\rho$ mass density), the non-constant impedance of the specimen $z_{s}$, and the constant impedance of the output bar $z_{o}$. The presence of any other impedances implies unaccounted reflection and transmission of the elastic waves and causes errors in the results. These errors typically manifest themselves as oscillations or broadening of peaks in the resulting stress/strain curves.

Given this preamble, a complication for an SHTB experiment arises in practice because the specimen needs to be securely attached to the input and output bars without introducing significant changes in the acoustic impedance. In the case of cylindrical specimens, this may easily be achieved by machining an exterior thread on the specimen and an interior thread into the bar ends. The specimen is then threaded into the bar ends and a lock nut is used to pre-tension the threads [5]. However, not all materials can be machined to a cylindrical shape but are instead available as sheets only. Some materials, e.g. laminated sheets of fiber-reinforced plastics, or rolled steel sheets are produced and used in their sheet form, demanding that they are tested in their intrinsic thin plate shape.

To attach sheet type specimens to the input and out bars, two methods are reported in the literature. The first method relies on cutting a slot into the bar which is slightly larger than the specimen thickness, and bonding the specimen into the slot with high-strength adhesives such as epoxy resins. Adhesive bonding has been proven to work well for both metals and plastic which react well to adhesive bonding, e.g. epoxy-based fibre-reinforced plastics. As this method adds only a very small contribution of an- 
other component into the above listed trinity of input bar, specimen, and output bar impedances, the resulting stressstrain curves can be highly accurate. The drawback of adhesive bonding is, however, that it is very labor-intensive to perform and can only be used for materials that can be easily bonded.

The second method relies on mechanically clamping the specimen, using either form-fit or friction based approaches, or combinations thereof [6]. In the special case that the width of the specimen tab, i.e., the area where it is gripped, does not exceed the bar diameter, it is sufficient to slot the bar and insert the specimen into the bar. A bolt through both specimen and slotted bar is then used to clamp down the arrangement and provide sufficient friction. This method also performs well, because, apart from the bolt, no components with different acoustic impedance are introduced. The approach has been successfully used for metal specimens [7] but is somewhat restricted to small specimens because the clamping surface that transfers friction forces is limited by the bar diameter, and the parallel gauge section of the specimen needs to be substantially smaller in width than the specimen tabs.

Attempts to circumvent this restriction by using larger clamping devices have been only weakly successful so far, at least to the knowledge of these authors. The reason is that such clamping devices typically introduce additional mass into the system, which changes the acoustic impedance and violates the assumptions of the SHTB experiment: Even if the same material as the bar material is used, the increased cross section area of such a device directly affects the impedance. This causes elastic wave transmission and reflections to occur at the interface between bars and clamping device, and these waves lead to artifacts in the stress-strain diagram.

In this paper, we address the problem of designing a mechanical clamping device for the SHTB which allows to test specimens with tabs larger than the bar diameter and features the same acoustic impedance as the input and output bars. We realize this by solving a mathematical statement of constant acoustic impedance for a possible clamping device geometry.

The benefits of our new clamping device are demonstrated by reporting tensile stress-strain data for Polycarbonate at high strain rates. Compared to other published high strain-rate tensile data, the new results are devoid of oscillations, even in the initial elastic part of the stressstrain diagram.

\section{Design of a clamping device with constant acoustic impedance}

The new constant impedance clamping device is designed according to a compact set of constraints. The clamping device material is chosen to be the same as the bar material -7075 grade aluminum. This choice fixes the speed of sound $c$ and the mass density $\rho$, leaving only the cross section area as a free design parameter. The clamping device geometry needs to make a smooth transition from the circular cross section of the bar to a rectangular cross section

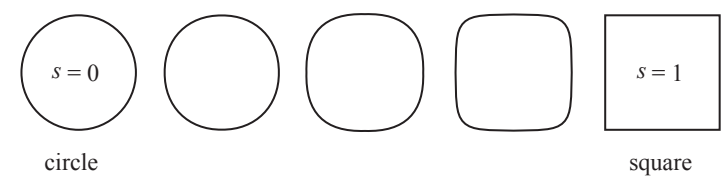

Fig. 1. Fernandez-Guasti squircles describe a smooth transition between squares and circles according to a squareness parameter $s$.

suitable to grip the flat specimen. We divide this task into two steps: (i) a smooth transition from circular to quadratic and (ii) a smooth transition from quadratic to rectangular. The first transition is achieved by employing FernandezGuasti squircles [8], a parametric representation of a continuous interpolation between circle and square.

$$
x^{2}+y^{2}-\frac{s^{2}}{r^{2}} x^{2} y^{2}=r^{2}
$$

The squareness parameter $s \in[0,1]$ interpolates between a perfect square and a perfect circle. To maintain a constant area, the parameter $r$ is adjusted. A visual representation of such squircles is shown in Fig. 1. In the CAD design of the clamping device, ten squircles are evenly spaced over a distance of $20 \mathrm{~mm}$ to effect the transition from circular to square. Similarly, a further ten rectangles are evenly spaced over $20 \mathrm{~mm}$ to effect a transition from square (aspect ratio 1:1) to a rectangle with aspect ratio of $\approx 2.5$. The final CAD design is shown in Fig. 2. This mount can clamp specimens of thickness $3 \mathrm{~mm}$. Clamping is achieved using four ISO M4 bolts, made of titanium to minimize additional mass. Each bolt provides a normal force of $4 \mathrm{kN}$ at a nominal tightening torque of $3 \mathrm{Nm}$. The total clamping force (two tab faces and four bolts) is thus at most $32 \mathrm{kN}$. The useful friction force acting on the specimen is a function of the friction coefficient $\mu$ between the aluminum mount and the specimen material. For typical polymers, for which this mount has been designed, $0.1<\mu<0.4$, such that maximum tensile forces of $3-15$ $\mathrm{kN}$ can be achieved.

\section{Experimental validation}

To assess the suitability of the new constant acoustic impedance clamping device, the stress-strain behavior of Polycarbonate at elevated rates of strain is investigated. We employ the SHTB setup described in [2], which uses aluminum bars of $16 \mathrm{~mm}$ diameter and a striker of 3000 mm length. This setup features a long pulse duration of $\approx 1.2 \mathrm{~ms}$ which makes it well suited to test materials such as PC with a very large strain to failure. The force acting on the specimen is inferred from conventional metal foil strain gauges on the output bar only. An amplifier with a bandwidth of $1 \mathrm{MHz}$ transforms the signal which is oversampled at $10 \mathrm{MHz}$ and digitized at 16 bit resolution. Engineering strain is measured locally on the specimen using a line scan camera which tracks the elongation of the parallel gauge section using two contrast marks, painted with an Edding 750 felt pen, at a rate of $200 \mathrm{kHz}$. The discrete 


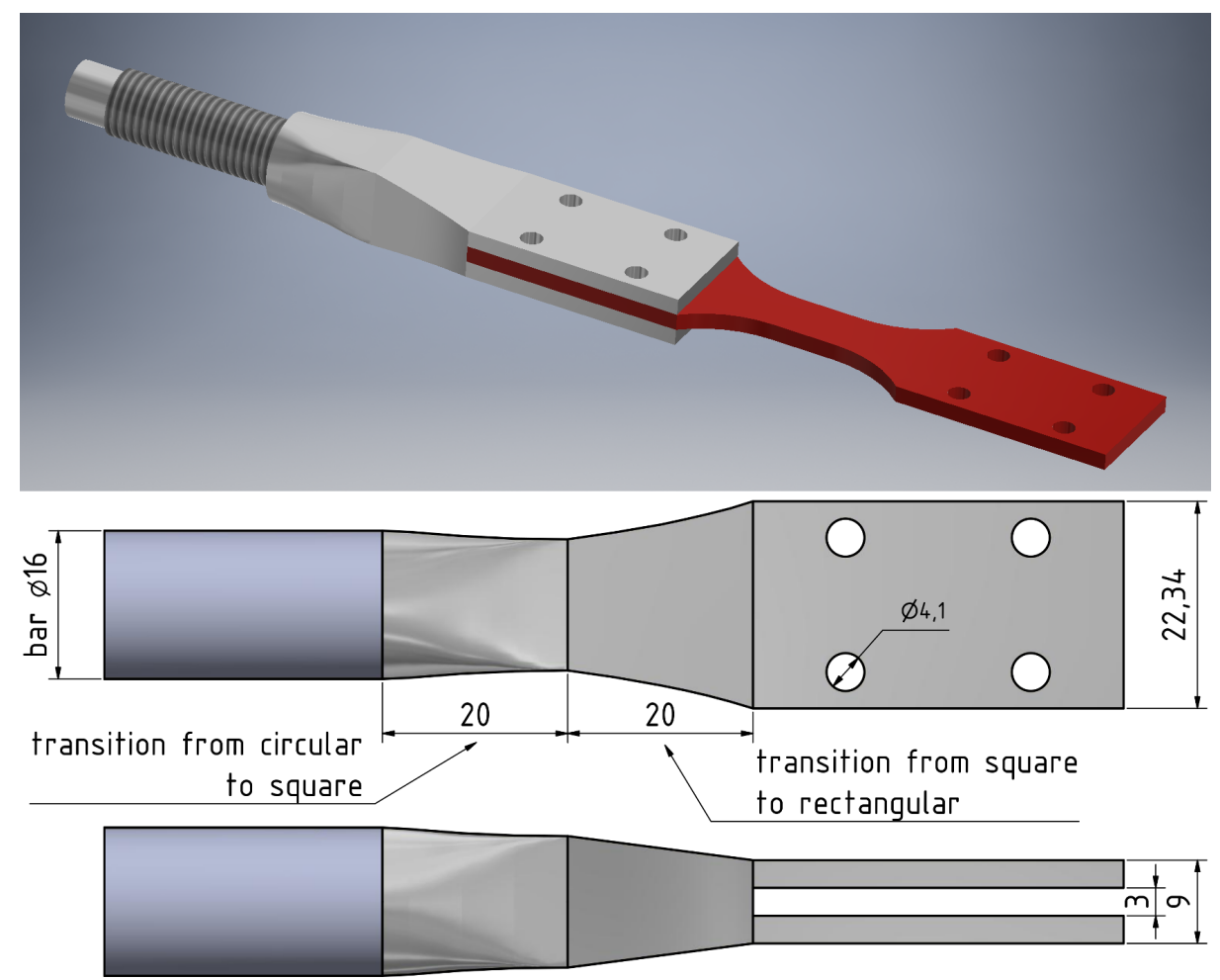

Fig. 2. upper part: CAD rendering of the constant acoustic impedance mount and a typical specimen. Only one mount is shown and clamping bolts a not displayed. The mount threads into the bar via an ISO M12x1.5 thread. Lower part: dimensioned drawing of the mount.

time events when images are acquired are also recorded along with the force data. In a post-processing step, the strain and force time series data are time-shifted in a deterministic way, taking into account the known distance between strain measurement position and force measurement position and the speed of sound in aluminium. The final result of this process is a nominal stress / nominal strain curve.

Specimens were machined from an extruded sheet of $3 \mathrm{~mm}$ thickness Polycarbonate (Makroclear ${ }^{\mathrm{TM}}$, Arla Plast Sweden). The specimen geometry with a nominal gauge length of $6 \mathrm{~mm}$ and cross section area of $30 \mathrm{~mm}^{2}$ is depicted in Fig. 3. We note that the $45^{\circ}$ circular transition from grip tab are to parallel gauge section has proven itself in numerous SHTB tests for various polymers as an optimal geometry to achieve a homogeneous deformation within the gauge section. However, due to the very smooth reduction of cross section, the effective specimen strain must be measured locally and a conventional calculation of strain using the reflected waves [9] is far from accurate.

A total of six specimens was tested at a striker velocity of $\approx 8 \mathrm{~m} / \mathrm{s}$, effecting a locally measured nominal strain rate of $1150 \mathrm{~s}^{-1}$. Specimen failure is achieved after a time of $\approx 0.8 \mathrm{~ms}$ due to the large failure strain in excess of $100 \%$. Fig. 4 shows the resulting individual nominal stress / nominal strain curves and an average of all curves. The individual curves are not smoothed in any way to give an accurate impression of the degree of quality achievable with this setup. Due to high degree of reproducibility, the individual data sets overlap and appear as one. The elastic regime

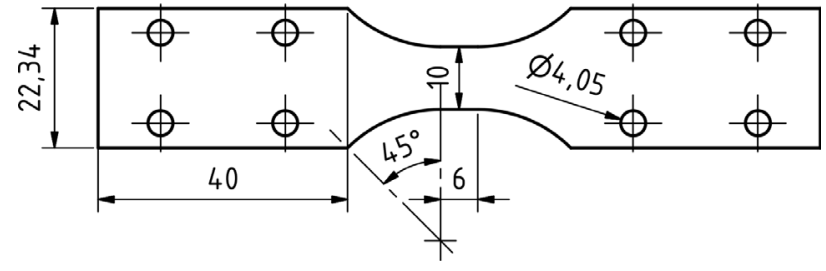

Fig. 3. Dimensioned drawing of the specimen used in this work, all dimensions in $\mathrm{mm}$.

of the averaged curve exhibits no visible amount of oscillations. Determination of the stiffness by means of linear regression within the strain range of $0.025 \%-0.5 \%$ yields a Young's modulus of $E_{d y n}=3.01 \pm 0.01 \mathrm{GPa}$. We are aware of the difficulties associated with the determination of dynamic moduli, nevertheless, this value compares favorably to the quasi-static Young's modulus of $E_{Q S}=2.46 \pm 0.01$ $\mathrm{GPa}$, assuming viscoelastic effects as also reported in [10]. The upper yield strain is resolved very well and the observed value agrees with the viscoplastic relationships reported in $[2,10]$.

\section{Conclusions}

In this paper, we report a novel design for mechanical clamping devices which can be used to test sheettype specimens in the Split-Hopkinson Tension Bar. Our Ansatz is to eliminate oscillations in the stress-strain curves that are observed in this experiment at their root. 


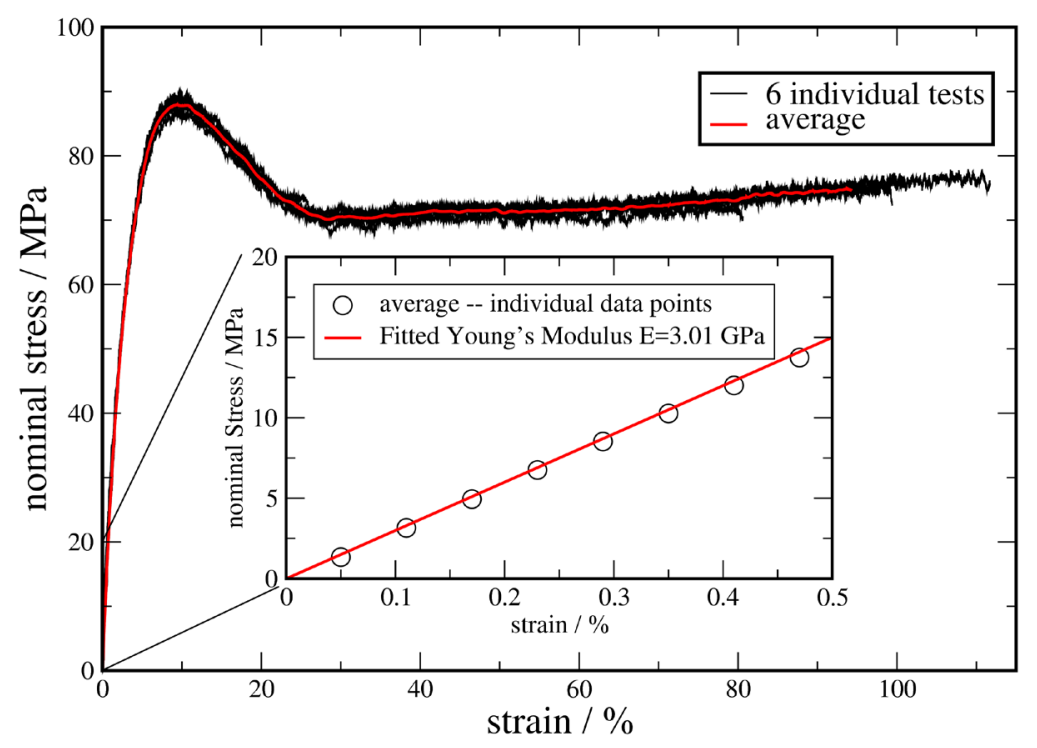

Fig. 4. Stress-strain diagram for Polycarbonate at a strain rate of $1150 \mathrm{~s}^{-1}$. Black curves display the individual data for a total of 6 different specimens. The red curve represents an average of all individual specimen data. Insert: A closeup of the elastic regime showing the individual data points of the average curve and a fit to the Young's modulus in the strain range $0.025 \%-0.5 \%$.

We do so by designing the clamping device in such a way that the acoustic impedance of the system is not modified. To this end, we implement a mathematical statement of constant acoustic impedance in the design process of the clamping device. The suitability of the resulting clamping device for high-strain rate tests is assessed by performing tests using flat specimens machined from sheets of Polycarbonate. We find that the quality of the resulting stressstrain curves is sufficient to accurately observe viscoelastic and viscoplastic effects. It is therefore expected that our new design provides a useful tool for practitioners of the SHTB method, as it allows to test sheet specimens directly and without laborious adhesive bonding work.

\section{References}

1. K.T. Ramesh, in Springer Handbook of Experimental Solid Mechanics (Springer, Boston, MA, 2008), pp. 929-960
2. G.C. Ganzenmüller, E. Blaum, D. Mohrmann, T. Langhof, D. Plappert, N. Ledford, H. Paul, S. Hiermaier, Procedia Engineering 197, 109 (2017)

3. R. Gerlach, C. Kettenbeil, N. Petrinic, International Journal of Impact Engineering 50, 63 (2012)

4. E. Mancini, M. Sasso, M. Rossi, G. Chiappini, G. Newaz, D. Amodio, J. Dynamic Behavior Mater. 1, 201 (2015)

5. B. Song, P.E. Wakeland, M. Furnish, Journal of Dynamic Behavior of Materials 1, 153 (2015)

6. N. Ledford, H. Paul, G. Ganzenmüller, M. May, M. Höfemann, M. Otto, N. Petrinic, EPJ Web of Conferences 94, 01049 (2015)

7. R. Smerd, S. Winkler, C. Salisbury, M. Worswick, D. Lloyd, M. Finn, International Journal of Impact Engineering 32, 541 (2005)

8. C. Fong, arXiv:1509.06344 [math] (2015)

9. M.A. Meyers, Dynamic Behavior of Materials (John Wiley \& Sons, 1994)

10. K. Cao, X. Ma, B. Zhang, Y. Wang, Y. Wang, Materials Science and Engineering: A 527, 4056 (2010) 